\title{
Segregación de la política fiscal en el esquema de metas de inflación ${ }^{1}$
}

\author{
Fiscal Policy segregation in the inflation targeting scheme
}

\author{
Josefina León León* \\ Juan Carlos Hurtado Ramos** \\ César D. Alvarado Gutiérrez ${ }^{* * *}$
}

\section{RESUMEN}

La política fiscal gozó de gran popularidad durante el periodo de posguerra como principal herramienta de la estabilidad macroeconómica. Con el paso del tiempo comenzó a perder relevancia, quedando relegada sólo para estabilizar los ciclos. El artículo tiene como objetivo revisar por qué la política fiscal ha sido relegada a un segundo plano dentro de los análisis teóricos y analizar su papel implícito en el Nuevo Consenso Monetario. Si bien es cierto que la política monetaria ha sido la

\footnotetext{
*Economista, Universidad de Guadalajara, Jalisco, México. Maestra en Políticas Públicas, Instituto Tecnológico Autónomo de México, México. Doctora en Ciencias Económicas, UAM, México. Profesora-Investigadora, responsable del proyecto de investigación, Departamento de Economía de la UAM Unidad Azcapotzalco, Av. San Pablo 180, Colonia Reynosa Tamaulipas, Delegación Azcapotzalco, Ciudad de México, C.P. 022000. México. Teléfono: 01-55-44376774. Correo-e: leon2josefita@hotmail.com

**Economista, UAM Unidad Azcapotzalco, Ciudad de México, México. Maestro en Ciencias Económicas, UAM, México. Estudiante del Doctorado en Ciencias Económicas, UAM, México. Teléfono: 55-44-58-40-69.

Correo-e: hurtadoramos@outlook.com

***Economista, Benemérita Universidad Autónoma de Puebla, Puebla, México. Maestro en Ciencias Económicas, UAM, México. Doctor en Ciencias Económicas, UAM, México. Profesor-Investigador, pertenece al Grupo de Investigación de Macroeconomía Financiera, Departamento de Economía de la UAM Unidad Azcapotzalco. Teléfono: 5-318-91-31. Correo-e: cdag@azc.uam.mx

1 Artículo de investigación científica que se origina por la necesidad de entender el papel de la política fiscal de una manera explícita dentro del esquema del Nuevo Consenso Monetario. Grupo de investigación de Macroeconomía Financiera. Nombre del proyecto de investigación: Teoría y política monetaria: evaluación y perspectivas. Universidad Autónoma Metropolitana (UAM).
}

(C) Los autores. Este artículo es publicado por Pensamiento Crítico de la Facultad de Ciencias Económicas, Universidad Nacional Mayor de San Marcos. Este es un artículo de acceso abierto, distribuido bajo los términos de la licencia Creative Commons Atribucion - No Comercia_Compartir Igual 4.0 Internacional. (http://creativecommons.org/licenses/by-nc-sa/4.0/) que permite el uso no comercial, distribución y reproducción en cualquier medio, siempre que la obra original sea debidamente citada. 
encargada de crear las condiciones necesarias para generar estabilidad, la política fiscal resulta crucial para alcanzar el objetivo sobre la tasa de inflación.

Palabras Clave: Política monetaria; coordinación de políticas; política fiscal; deuda. Clasificación JEL: E52, E61, E62, H63

\section{ABSTRACT}

Fiscal policy enjoyed great popularity during the post-war period as the main tool of macroeconomic stability. With the passage of time it began to lose relevance, being relegated only to stabilize the cycles. The objective of this paper is to review why fiscal policy has been relegated to a second place within theoretical analyses and to analyze its "implicit" role in the New Macroeconomic Consensus. While it is true that monetary policy has been responsible for creating the necessary conditions to generate stability, fiscal policy is crucial to reach the objective on the inflation rate.

Palabras Clave: Monetary politics; policy coordination; fiscal policy; debt.

Clasificación JEL: E52, E61, E62, H63

\section{Introducción}

La política fiscal gozó de gran popularidad durante el periodo de posguerra como principal herramienta de la estabilidad macroeconómica, pero con el paso del tiempo comenzó a perder relevancia, quedando relegada sólo para estabilizar los ciclos. En la década de los setenta se empieza a perfilar la política monetaria como eje conductor de la estabilidad, la importancia del dinero fue señalada por la corriente monetarista encabezada por Milton Friedman, autor que considera que las variaciones en la cantidad de dinero afectan en el corto plazo tanto a variables nominales como a variables reales.

Bajo este análisis se deja de lado el planteamiento de Jan Tinbergen, ${ }^{2}$ de que para cada objetivo de política económica se tenía que utilizar un instrumento, en su lugar se plantea que un solo instrumento, en este caso el control adecuado de la oferta monetaria a cargo del Banco Central (BC), puede lograr 3 objetivos: estabilidad de precios, crecimiento económico y generación de empleo. Además, en el marco de las aportaciones de la nueva escuela clásica comienza una tendencia a favor del uso de reglas y en

2 Uno de los pioneros en la aplicación de herramientas matemáticas a la economía. Galardonado, en conjunto con RagnarFrisch en 1969, con el Premio Nobel de Economía. 
contra de medidas discrecionales, al considerar que las reglas aumentan la eficiencia en la toma de decisiones del sector público.

Este enfoque que defiende la filosofía del neoliberalismo económico se convierte en una fuente de inspiración para posteriores análisis críticos que desembocaron en la corriente de los nuevos keynesianos. La nueva escuela clásica y la nueva economía keynesiana influyen en la Nueva Síntesis Neoclásica, corriente en la que se desarrolla el esquema de metas de inflación (EMI), donde la política monetaria desempeña un papel hegemónico y la política fiscal un papel pasivo. Por lo tanto, en la macroeconomía ortodoxa se hace referencia al nuevo paradigma monetario o Nuevo Consenso Monetario (NCM), ya que el BC se encarga de generar las condiciones de estabilidad a través de modificar la tasa de interés nominal de corto plazo.

Hanel y Rosenthal(1997, p. 4) afirman que el BC tiene dos opciones de objetivos para evitar la variación de la tasa de inflación: objetivos directos $^{3}$ y objetivos intermedios ${ }^{4}$. estos últimos los clasifica en: agregados monetarios, tipo de cambio y tasa de interés. A la lista se pueden agregar las expectativas de inflación, las cuales actúan (implícita o explícitamente) como un objetivo intermedio de política monetaria en el esquema de metas de inflación (EMI), ya que las decisiones de las autoridades monetarias suelen estar guiadas por la desviación de los pronósticos de inflación futura respecto de la inflación anunciada como meta en un horizonte de mediano plazo, de acuerdo con la regla de Taylor (1993). ${ }^{5}$

Para lograr sus objetivos la autoridad monetaria debe operar de manera autónoma ${ }^{6}$ generando confianza y credibilidad en su comunicación

\footnotetext{
3 Hanel y Rosenthal(1997, p. 4) definen a los objetivos intermedios como las metas de inflación. El BC tendrá una serie de herramientas de política monetaria, como lo son los agregados monetarios, el tipo de cambio y la tasa de interés, utilizando las que crea pertinentes para lograr el objetivo de estabilidad en el nivel de precios.

4 Los objetivos intermedios dentro de la literatura académica se deben entender como instrumentos de política monetaria para mantener la estabilidad en el nivel de precios.

5 La regla de Taylor se puede considerar como un marco de referencia en el que el BC utiliza a la tasa de interés de corto plazo como instrumento principal y establece como objetivos, la estabilidad de precios y el nivel de producción. Dicho marco se puede sintetizar en la siguiente expresión: $i_{t}=R+\pi^{*}+\alpha\left(Y-Y^{*}\right)+\beta\left(\pi_{t}-\pi^{*}\right)$. Donde $i_{t}$ es la tasa de interés nominal de corto plazo; $R$ es la tasa de interés real; $\pi^{*}$ es la inflación objetivo establecida por el BC; $\pi_{t}$ es la tasa de inflación observada; $\left(Y_{t}-Y^{*}\right)$ es la desviación del producto observado respecto a su nivel potencial; finalmente, $\alpha$, $\beta$ son parámetros positivos que representan las preferencias del BC.

6 De acuerdo al New Palgrave Dictionary of Economics, la independencia del BC se refiere a"la libertad que tienen los hacedores de política económica para ejercer de manera directa influencia política o gubernamental en la conducción de las políticas"[Traducción propia](Walsh, 2008, p. 366).
} 
con los mercados. ${ }^{7}$ La importancia de la política monetaria es incuestionable, pero ¿dónde queda el papel de la política fiscal?, ¿cuál es su comportamiento bajo el NCM? ¿El manejo sólo de la política monetaria garantiza la estabilidad económica?

El artículo tiene como objetivo revisar por qué la política fiscal ha sido relegada a un segundo plano dentro de los análisis teóricos y analizar su papel "implícito" en el NCM, puesto que, si bien es cierto que la política monetaria ha sido la encargada de crear las condiciones necesarias para generar estabilidad, la política fiscal resulta crucial para alcanzar el objetivo de inflación.

El trabajo se organiza como sigue: la primera parte corresponde a esta introducción, en la segunda se hace una breve revisión histórica sobre la evolución de la política fiscal en diferentes corrientes de pensamiento, señalando en una primera etapa su auge y su posterior segregación en el análisis de la macroeconomía ortodoxa. En la tercera sección se hace una revisión teórica del EMI con base en Jones (2009), donde juega un papel fundamental la política monetaria pero se evidencia la falta de incorporación de la política fiscal. En la cuarta parte se revisa el comportamiento de la política fiscal bajo el esquema de metas inflación considerando el artículo de Bai y Leeper(2017), autores que señalan que la coordinación entre ambas políticas garantiza el cumplimiento de la estabilidad de precios.

\section{Auge y ocaso de la política fiscal}

\subsection{Una nueva concepción: Keynes y los keynesianos}

Con el crac del 29 y la Gran Depresión se puso en entredicho a la teoría clásica dadas sus limitaciones y escasas explicaciones ante la serie de fenómenos que ocurrieron, cuestionando las ideas del laissez faire, laissez passer, como las más propicias para encaminar a las economías a la senda de la estabilidad.

La publicación de la Teoría General (1936) de Keynes generó una revolución en el pensamiento económico debido a la nueva forma de enfrentar los problemas económicos, lo que iba en contra de las ideas clásicas,

7 Véase Blinder (2000, p. 165). 
basadas en el análisis microeconómico asociado con la competencia perfecta y el mecanismo sancionador del mercado, dando como resultado la convergencia al equilibrio sin la ayuda de la intervención del Estado. Keynes cuestiona si el mercado bajo las ideas de la ley de Say ${ }^{8} \operatorname{logra}$ la convergencia al equilibrio de manera estable, el estancamiento con deflación de los años treinta era una muestra de que no era así, por lo tanto, propone la intervención del Estado a través de la política económica para ayudar a las economías a salir del estancamiento económico y no ser simplemente un ente observador.

La novedad de Keynes, no sólo se limitó a los conceptos que introdujo en su análisis, como la función consumo, la noción del multiplicador, la propensión marginal a consumir o la preferencia por la liquidez, sino, como afirma Blaug (2001, p. 754), lo nuevo es:

[...] la tendencia a trabajar con agregados y en efecto a reducir toda la economía a cuatro mercados interrelacionados de bienes, mano de obra, dinero y de bonos. En segundo lugar, la concentración en el corto plazo, de modo que el análisis de largo plazo, que había sido el principal foco analítico de sus antecesores, queda confinado a observaciones marginales sobre la probabilidad del estancamiento secular, y en tercer lugar la asignación de todo el peso de los ajustes ante el cambio de las condiciones económicas al nivel de la producción antes que a los precios.

De tal suerte que, la economía no converge de manera estable al equilibrio, sino que aparece un concepto que será la base de posteriores análisis teóricos: el equilibrio con desempleo ${ }^{9}$. Este planteamiento va en contra de la Ley de Say, dando como resultado el hecho de que las economías capitalistas enfrentan problemas de insuficiencia de demanda efectiva. ${ }^{10}$

Las ideas de Keynes tuvieron un fuerte impacto en una nueva generación de economistas que desarrollaron a partir de sus planteamientos una corriente de pensamiento que denominaron keynesianismo, cuyos autores defienden la intervención del Estado en la economía.

\footnotetext{
8 Para Keynes (1936) [2014], la Ley de Say, bajo el enfoque clásico es: "Según la cual el precio de la demanda agregada de la producción en conjunto es igual al precio de la oferta agregada para cualquier volumen de producción, equivale a decir que no existe obstáculo para la ocupación plena" (p. 57).

9 Por equilibrio con desempleo, se debe entender en la que todos los mercados se encuentran en equilibrio exceptuando el mercado de trabajo

10 La demanda efectiva es el punto de intersección entre la demanda global y la oferta global. Al existir desempleo, disminuye el ingreso de los trabajadores, con lo que se reduce el gasto. Por lo tanto, existe una sobreacumulación de bienes que no podrá ser absorbida por la economía, lo que conlleva a un círculo vicioso al alargar la situación de desempleo, de ahí la importancia de la intervención del Estado.
} 
Sus desarrollos se interpretan y sintetizan en lo que se conoce como la síntesis neoclásica (SN), ${ }^{11}$ dando como resultado que durante el periodo de 1940 a 1960 hubo un fuerte interés por el estudio del modelo del multiplicador ${ }^{12}$ que analiza de forma minuciosa los efectos provocados en la economía por el uso tanto de la política fiscal como monetaria, siendo el multiplicador de la política fiscal el que cobró mayor relevancia ${ }^{13}$. La política monetaria se consideraba como un complemento para la política fiscal, dicha política debía encargarse de mantener baja la tasa de interés con el fin de reducir el costo de la deuda pública.

Abba Lerner (1944) justifica de manera directa el uso a voluntad del déficit por parte del Estado, considera que este no debe seguir una tendencia de finanzas sanas considerado como prudencia fiscal, sino apoyarse en las finanzas funcionales donde la variación del déficit debe de tener un manejo práctico dependiendo de la situación del ciclo económico para así poder cumplir sus objetivos. El concepto de finanzas públicas funcionales va en contra de la idea de finanzas sólidas o de un presupuesto equilibrado, como mencionan Snowdon y Vane (2002, p. 286).

En el caso de la regla de equilibrio presupuestario, un gobierno se vería obligado a reducir sus gastos y/o aumentar los impuestos durante una recesión (ya que los ingresos tributarios disminuyen a medida que el producto interno bruto (PIB) disminuye) y a aumentar los gastos públicos durante un período de auge (ya que los ingresos tributarios aumentan durante un período de auge). Desde una perspectiva keynesiana, estas acciones desestabilizarían aún más una economía ya inestable; es decir, estabilizar las finanzas desestabiliza la economía. El principio de las finanzas

\footnotetext{
11 Este movimiento intelectual, comenzó con la publicación del artículo de J.R. Hicks (1937) Mr. Keynes and the "Classics"; A SuggestedInterpretation,que plantea el modelo $I S$ - $L M$, culminando con su popularización por parte de Paul Samuelson (1947), Foundations of EconomicAnalysis, dentro de los Estados Unidos y el mundo académico en general. 12 La idea del multiplicador fue desarrollada por el economista inglés Richard Kahn, en su artículo de 1931, The Relation of Home Investment to Unemployment, que inspiró al propio Keynes en la construcción de su multiplicador de la inversión. La noción del trabajo de Kahn muestra que un aumento en la inversión pública conlleva a un aumento en el empleo total de la economía, siendo este un fuerte instrumento en la lucha por disminuir los niveles de desempleo que aquejaban al período de la Gran Depresión. El modelo del multiplicador, en su conjunto contiene el análisis del: $a$ ) multiplicador de las compras del Estado, b) el multiplicador de los impuestos y c) el multiplicador monetario. En los análisis keynesianos, el multiplicador del dinero se mantiene fijo.

13 En el modelo keynesiano el dinero es no neutral, pero la política monetaria puede ser ineficaz para afectar los niveles de producción y empleo particularmente en dos casos especiales: i) la trampa de liquidez, la cual se asocia con una situación de recesión económica donde el nivel de la tasa de interés es muy bajo o cercano a cero y existe incertidumbre sobre el futuro, por lo tanto un aumento en la oferta monetaria no impulsa a la economía, dado que los agentes económicos prefieren guardar el dinero en su casa y no comprar bonos que rinden una tasa de interés casi nula, por lo que la tasa de interés ya no puede bajar más y en consecuencia la política monetaria es ineficaz. ii) La insensibilidad de la inversión ante variaciones en la tasa de interés, en este caso la política monetaria expansiva provoca una disminución en la tasa de interés, pero la inversión se mantiene fija. Por lo tanto, en ambas situaciones la política fiscal será la única herramienta para impulsar el crecimiento de la economía.
} 
funcionales sugiere que el gobierno debe equilibrar su presupuesto a lo largo del ciclo, es decir, tener déficit durante las recesiones y superávit durante los períodos de auge. Al hacerlo, el gobierno ayudará a reducir la severidad de las fluctuaciones agregadas. ${ }^{14}$

De acuerdo con Lerner, la magnitud del déficit se convierte en irrelevante cuando se trata de alcanzar los objetivos macroeconómicos de crecimiento económico, generación de empleo y estabilidad de precios, pero estas ideas no fueron del todo bien recibidas e incluso hubo críticas por parte de algunos autores keynesianos. ${ }^{15}$

A principios de la década de los setenta se empieza a romper el consenso keynesiano debido a la estanflación económica que se genera, un problema que no tenía explicación hasta el momento, dado que el modelo IS-LM y la curva de Phillips explican una situación de recesión con deflación o expansión con inflación. Por lo tanto, las ideas keynesianas empezaron a ser cuestionadas, aunado a la ausencia de microfundamentos.

\subsection{Cambio de paradigma: hacia el libre mercado}

Lucas (1972), formuló uno de los primeros modelos con microfundamentos, en el cual explica que los ciclos económicos están asociados a cambios no anticipados en la oferta monetaria. Bajo esta misma lógica, pero para la política fiscal, Barro (1974),argumenta que una disminución de los impuestos no incrementará el nivel de actividad económica. La disminución de los impuestos generará una reducción en los ingresos del Estado, este faltante de ingresos será cubierto mediante deuda, por tanto, los agentes no modificarán su gasto pues saben, que esta deuda será pagada con impuestos futuros. Dando como resultado, que cualquier intento del Estado por afectar la demanda agregada por medio de políticas fiscales expansivas estará destinado a fracasar, lo que contradice la idea del multiplicador keynesiano.

Por otro lado Kydland y Prescott (1977)desarrollan un modelo que incorpora aspectos como la hipótesis de expectativas racionales, la curva

14 Traducción propia.

15 La idea viene de la cita que hacen Landreth y Colander(2006) al pie de página a partir de una entrevista hecha por los mismos autores a Evsey Domar: "Incluso los keynesianos, tras oír el argumento de Lerner de que la magnitud del déficit no era importante, se revolvieron y dijeron: no, está equivocado, no cabe ninguna duda" (p. 426). 
de oferta de Lucas ${ }^{16}$ y la teoría de juegos, con estos fundamentos muestran que la política monetaria discrecional es inconsistente en el tiempo. En consecuencia, los autores recomiendan que las decisiones de política monetaria se deben sujetar a una regla con el fin de que la economía logre un equilibrio óptimo.

Con estas ideas empieza a dominar desde la década de los setenta la filosofía del neoliberalismo económico, las críticas por parte de los autores de la nueva escuela keynesiana no se hicieron esperar; Mankiw y Romer (1991) señalan que en la nueva perspectiva keynesiana las fluctuaciones económicas se pueden explicar con rigurosos modelos microeconómicos asociados con la existencia de fallas de mercado: costos en el ajuste de precios, competencia imperfecta, existencia de contratos salariales traslapados y fallas de coordinación, lo que abre paso al papel de la política económica, sobre todo de la política monetaria en la que se empieza a gestar la estrategia de metas de inflación. ${ }^{17}$

\section{Auge y ocaso de la política fiscal}

De acuerdo con Roger (2010, p. 46) la evidencia teórica y empírica ha mostrado que mantener una tasa de inflación baja y estable ayuda al mejor desempeño de las economías en el largo plazo, por tanto, la búsqueda de la estabilidad sobre esta tasa es uno de los ejes centrales en los actuales análisis teóricos y para dicho propósito el encargado será el BC. ${ }^{18}$

Durante la década de los años noventa, el uso de la tasa de interés de mercado se convirtió en la herramienta primordial para lograr dicho propósito, como señalan García y Perrotini (2014, p. 37) "Así, en los años noventa cristalizó un doble consenso, primero, en torno de los beneficios que una macroeconomía de baja inflación representa para el crecimiento económico, el empleo y la maximización del bienestar y, segundo, acerca de la

\footnotetext{
16 La cual asume que los choques monetarios imprevistos son la causa principal de la inestabilidad agregada.

17 Según el Annual Report on Exchange Arrangements and Exchange Restrictions (2017, p. 6-7), publicado por el Fondo Monetario Internacional los países que actualmente cuentan con un EMI como marco principal de política económica son 40: Albania, Armenia, Australia, Brasil, Canadá, Chile, Colombia, Corea del Sur, Filipinas, Georgia, Ghana, Guatemala, Hungría, India, Indonesia, Islandia,Israel, Japón, Kazajistán, México, Moldavia, Noruega, Nueva Zelanda, Paraguay, Perú, Polonia,Reino Unido, República Checa, República Dominicana, Rumania, Rusia, Serbia, Sudáfrica, Suecia,Tailandia, Turquía, Ucrania, Uganda, Uruguay. Argentina adoptó este marco de política monetaria en el año 2016 para abandonarlo durante el primer cuatrimestre de 2018.
}

18 Véase Hetzel (2004, p.47). 
tasa de interés como reguladora de los precios para alcanzar una meta de inflación baja y estable".

Antes de llegar a dicho consenso, se da un debilitamiento en torno a la teoría monetarista que predominó de mediados de los setenta a la década de los ochenta. Una razón para que haya ocurrido este debilitamiento es atribuido a su propia construcción teórica, al estar fuertemente ligada a la teoría cuantitativa del dinero, que considera constante la velocidad de circulación del dinero, ante cualquier variación de esta variable afectará indiscutiblemente los resultados.

Esto se pudo observar durante la década de los ochenta, debido al fuerte cambio que sufrió el sistema financiero, el cual se atribuye a la desregulación financiera a nivel global, que se tradujo en una alta volatilidad en la velocidad de circulación del dinero dada por su alta sensibilidad a la tasa de interés. Además, los hacedores de política económica consideraron que mantener la estabilidad en la tasa de inflación por medio del control de los agregados monetarios era sumamente rígido, por lo cual optaron por otros instrumentos de política monetaria como el manejo de la tasa de interés nominal.

Paul Volcker, gobernador de la Reserva Federal (Fed), en 1979 aumentó la tasa de interés a niveles del $11.2 \%$, siendo el mismo año que la propia Fed anunció la trayectoria para el agregado monetario $\mathrm{M} 1^{19}$ y para el año de 1982 se dejó que la tasa de interés cayera. Para 1993, el gobernador en turno de la Fed, Alan Greenspan, anunció que el control de los agregados monetarios deja de ser la herramienta que conduce la política monetaria de Estados Unidos. Por lo tanto, inauguró el uso de la tasa de interés como herramienta principal de política monetaria siguiendo los criterios establecidos por la regla de Taylor (1993), lo anterior con la finalidad de lograr los objetivos de actividad económica y estabilidad de precios; para 1990 Nueva Zelanda comenzó con el uso del EMI como marco primordial de política económica, debido a que, el control de la tasa de interés permite una mayor flexibilidad a la hora de actuar y disminuir la fuerte presión sobre la tasa de inflación, como señala Bernanke y otros (2001, p.4) "El esquema de metas de inflación es un marco para la política monetaria que se caracteriza por el anuncio oficial al público de objetivos

19 Véase Meulendyke (1988, p.11). 
cuantitativos (o rangos de objetivos) de la tasa de inflación, en uno o más horizontes temporales y por el reconocimiento explícito de que una inflación baja y estable es el objetivo principal de la política monetaria"20.

\subsection{Un modelo sencillo de metas de inflación}

Esta sección analiza el modelo de tres ecuaciones propuesto en el marco del NCM, utilizamos la presentación sencilla que expone Jones (2009), en este sistema la dicotomía clásica no se cumple en el corto plazo y por lo tanto, las variables nominales afectan a las variables reales. ${ }^{21}$

El BC es el encargado de estabilizar la economía y esto lo logra a partir del manejo de la tasa de interés real, ${ }^{22}$ dado por un movimiento en la tasa de interés nominal, para influir directamente sobre el nivel de producción efectiva y como consecuencia sobre la tasa de inflación. Pero se deja de lado el papel de la política fiscal, que como veremos más adelante, tiene que influir para que se pueda cumplir la estabilidad en la tasa de inflación, dicho de otra forma, en este análisis no se observa la coordinación entre ambas políticas, mostrando sólo la importancia de la política monetaria.

Supuestos del modelo:

1. Una economía pequeña y cerrada.

2. Se hace distinción entre el corto plazo expresado como el nivel de producción efectiva $\left(Y_{t}\right)$ y el largo plazo expresado como el nivel de producción potencial al que tiende la economía $(\bar{Y})$.

20 Traducción propia.

21 Para una presentación matemática del modelo, ver por ejemplo el trabajo de Clarida, Galí y Gertler (1999).

22 Este sencillo modelo de metas de inflación asume movimientos en la tasa de interés real y rigidez de precios, pero como el $\mathrm{BC}$ no puede establecer de manera directa la tasa de interés real, por medio de la versión sencilla de la ecuación de Fisher se puede ver cómo reacciona la tasa de interés real ante variaciones en la tasa de interés nominal $R_{t}=i_{t}-\pi_{t}$ ante dos posibles escenarios:

- Como el modelo asume rigidez en la tasa de inflación, una variación en la tasa de interés nominal conduce a una variación directamente proporcional en la tasa de interés real, es decir $\Delta R_{t}=\Delta i_{t}$

- Si no se asumiera tal rigidez, la variación en la tasa de interés nominal tendría que ser mayor a la variación de la tasa de inflación, es decir $\Delta i_{t}>\Delta \pi_{t}$

En este modelo se supone que el BC mueve la tasa de interés nominal para afectar directamente a la tasa de interés real. 
3. La economía consta de 3 ecuaciones: ${ }^{23}$

- $\quad$ Curva IS $\Rightarrow Y_{t}=\bar{a}-\bar{b}\left(R_{t}-\bar{r}\right)$

Donde $\bar{a}$ es un factor de desplazamiento en el cual estarán incorporados todos los valores autónomos de la demanda agregada $(\bar{a}=\bar{C}+\bar{I}+G-1), \bar{b}$ es un parámetro de sensibilidad de la inversión, $R_{t}$ es la tasa de interés real, $\bar{r}$ es la productividad marginal del capital y $\left(R_{t}-\bar{r}\right)$ es la desviación de la tasa de interés real con respecto a la productividad marginal del capital.

Curva $\mathrm{PM} \Rightarrow$ Refleja como el BC, por medio de la variación en $R_{t}$ modifica tanto el nivel de producción efectiva, como la tasa de inflación efectiva $\pi_{t}$.

- $\quad$ Curva de Phillips $\Rightarrow \pi_{t}=\pi_{t-1}+\bar{v} Y_{t}+\bar{o}$

- $\quad$ Donde $\pi_{t-1}$ es la tasa de inflación de $t-1$ para el periodo $t, \bar{v}$ es un parámetro de sensibilidad de fijación de precios, $\bar{v} Y_{t}$ es la actividad económica y $\bar{o}$ es un choque en los precios. Se puede ver también que: $\Delta \pi=\pi_{t}-\pi_{t-1}$.

4. Los agentes poseen expectativas adaptativas ${ }^{24}$, es decir $\pi_{t}^{e}=\pi_{t-1}^{e}+\gamma\left(\pi_{t}-\pi_{t-1}^{e}\right)$ Donde $\pi_{t}^{e}$ es la tasa de inflación esperada para el periodo $\mathrm{t}, \pi_{t-1}^{e}$ es la tasa de inflación esperada en t-1, $\left(\pi_{t}-\pi_{t-1}^{e}\right)$ es la desviación de la tasa de inflación efectiva con respecto a tasa de inflación esperada en t-1 y $\gamma$ es un parámetro de ajuste parcial definido como un término de ajuste de error, donde $0<\gamma \leq 1$.

5. Existe rigidez en la tasa de inflación, entendiéndose como el lento ajuste de èstadurante el tiempo.

Ante esto, el BC tiene como propósito mantener la estabilidad en la tasa de inflación y lo hace a través de una regla de política monetaria la cual es un manual de acciones a seguir para lograr dicho objetivo.

23 Para una explicación detallada de la derivación de la curva IS, la curva PM y la Curva de Phillips, se pueden revisar los capítulos 9, 10 y 11 en Jones (2009, p. 321-450).

24 Se asume este tipo de expectativas para ver el desenvolvimiento de la economía ante un cambio del objetivo de la tasa de inflación $(\bar{\pi})$, pero en una versión más a detalle se incluyen las expectativas racionales. 
Una regla sencilla, que puede seguir el BC, es hacer que la tasa de interés real dependa de la tasa de inflación, como la siguiente:

$$
i_{t}-\bar{r}=\alpha\left(\pi_{t}-\bar{\pi}\right)
$$

Donde $\alpha$ es un parámetro de reacción del BC que determina la agresividad con la que se debe de manejar la política monetaria.

Partiendo de la ecuación (3) podemos sustituir la regla de política monetaria, representada por la ecuación (4), para obtener la curva de Demanda Agregada (DA).

$$
Y_{t}=\bar{a}-\bar{b}\left(i_{t}-\bar{r}\right)
$$

Se obtendrá:

$$
D A=\bar{a}-\bar{b}\left[\alpha\left(\pi_{t}-\bar{\pi}\right)\right]
$$

La ecuación (5) denota a la curva de DA. Esta curva tiene una pendiente negativa, debido a la respuesta de los hacedores de política económica en su objetivo por mantener la estabilidad sobre la tasa de inflación con base a la regla de política monetaria, es decir, ante desviaciones de la tasa de inflación con respecto a su objetivo y al existir una regla de política monetaria, el BC actuará de la siguiente forma:

1. Si $\pi_{t}>\bar{\pi} \Rightarrow$ El BC aumentará la tasa de interés $\left(i_{t}\right)$. Este aumento será mayor que la productividad marginal del capital $\bar{r}$ por lo que provoca una disminución en el nivel de inversión y por ende una disminución en el nivel de producción efectiva encontrándose ésta por debajo de su nivel potencial.

2. Si $\pi_{t}<\bar{\pi} \Rightarrow \mathrm{BC}$ disminuirá la tasa de interés $\left(i_{t}\right)$. Esta disminución será menor que la productividad marginal del capital $\vec{r}$. por lo que provoca un aumento en el nivel de inversión y por ende un aumento en el nivel de producción efectiva encontrándose ésta por encima de su nivel potencial.

La agresividad con la actúa el BC depende del parámetro $\alpha$, si éste es muy alto indica que el $\mathrm{BC}$ ante una mínima alza de la tasa de inflación tiene que reaccionar con una fuerte subida en la tasa de interés y viceversa. 
La curva de Oferta Agregada (OA) tendrá pendiente positiva y para tal fin se hace uso de la curva de Phillips aumentada con expectativas para su construcción, la cual está expresada en la ecuación (2):

$$
O A: \Delta \pi=+\bar{v} Y_{t}+\bar{o}
$$

La pendiente positiva de la OA indicará la conducta de las empresas en torno a la fijación de precios. Cuando $Y_{t}>\bar{Y}$, las empresas tratarán de mantener el nivel de producción que esté ligada al nivel de demanda en ese momento. Los precios tienden a aumentar debido al incremento en los costos de producción (como las horas extras o los costos de los factores de producción), por tanto, cuando todo el conjunto de empresas sube los precios se dará un aumento en la tasa de inflación. A raíz de esto se puede asumir una relación directa entre el nivel de producción efectiva $\left(Y_{t}\right)$ y la tasa de inflación $\left(\pi_{t}\right)$.

Una vez deducidas las curvas de DA y $\mathrm{OA}$, se puede encontrar el equilibrio entre el nivel de producción efectiva $\left(Y_{t}\right)$ y la tasa de inflación objetivo $(\bar{\pi})$. Con base en este modelo Jones (2009) analiza la dinámica entre el nivel de producción y la tasa de inflación y muestra cómo responde el BC cuando la inflación efectiva se desvía de su objetivo. Cualquiera que sea la desviación, va a implicar que en el corto plazo exista una brecha entre la producción efectiva con relación a su nivel potencial, por lo tanto, el BC tiene que intervenir modificando la tasa de interés nominal hasta que la economía se encuentre de nuevo en su nivel potencial.

Gran parte del ajuste del sistema depende de la formación de expectativas por parte de los agentes, el BC debe convencer a los agentes para que ajusten sus expectativas de inflación en la dirección deseada, lo que se traduce en una disminución de la brecha de inflación, y como resultado la economía transita de forma paulatina de nuevo al equilibrio de largo plazo, donde el nivel de producción efectiva es igual al nivel de producción potencial y la tasa de inflación efectiva es igual a la meta establecida.

En este modelo la política monetaria juega un papel fundamental, pero ¿dónde queda la función de la política fiscal? En la práctica debe de existir coordinación entre ambas políticas para asegurar que el objetivo del control de la tasa de inflación sea cumplido en su totalidad. En la siguiente sección se analiza cuál debe de ser el comportamiento de la políti- 
ca fiscal bajo este enfoque para que brinde un fuerte respaldo a la política monetaria.

\section{Política fiscal bajo un esquema de metas de inflación}

Como se mencionó anteriormente, Roger (2010) señala que la evidencia empírica muestra que los países que han adoptado estos esquemas han reducido la presión sobre la tasa de inflación, pero, también se ha comprobado que esta presión no disminuye únicamente por el movimiento de la tasa de interés, sino que la presión de la tasa de inflación disminuye siempre y cuando los agentes económicos tengan la expectativa de que va a disminuir, pero ¿dónde queda el papel de la política fiscal?

En la actualidad la literatura no es muy explícita respecto a cómo debe comportarse la política fiscal bajo este esquema, pero debe existir coordinación entre la política monetaria y la fiscal. Bajo esta perspectiva la palabra coordinación juega un papel relevante, ya que alcanzar el objetivo de estabilidad de precios sólo se cumple en función de dos factores:

1. Si la política monetaria es creíble acorde con el objetivo.

2. Si el déficit fiscal se mantiene en niveles bajos.

Por tanto, el objetivo de inflación se alcanza si se reduce, tanto la inflación como el déficit, es decir, ambas políticas deben ser acordes con el EMI.

Bai y Lepper (2017, p. 105) plantean que la política fiscal debe jugar un papel pasivo ${ }^{25}$, aunado a la importancia de las reglas de política fiscal, las cuales son indispensables para garantizar la sostenibilidad fiscal.

De acuerdo con Arenas (2016, p. 20) la sostenibilidad fiscal "no solo pertenece al ámbito de lo económico o lo fiscal, sino también es un punto clave de la sostenibilidad de las políticas públicas". Muchas decisiones en el campo de la política del sector público están fuertemente ligadas con la

\footnotetext{
25 Leeper (1991, p. 130) define a la política pasiva y activa como:"Una autoridad activa no presta ninguna atención al estado de la deuda pública y es libre de establecer su variable de control cuando lo considere oportuno. Una autoridad pasiva responde a las crisis de la deuda pública. Su comportamiento se ve limitado por la optimización privada y las acciones de la autoridad activa" [Traducción propia].
} 
tributación, y la recaudación de impuestos está vinculada con casi todos los aspectos económicos y sociales.

En la literatura económica pueden encontrarse diversas definiciones sobre la sostenibilidad fiscal. Bohn (1998, p.950) la define como "la política gubernamental es sostenible en el sentido de satisfacer una restricción presupuestaria intertemporal". Esto evita que los Estados tengan que endeudarse para financiar sus gastos y por consiguiente la deuda pública crezca de manera exponencial teniendo como consecuencia la incapacidad de las obligaciones por parte de los Estados. Arenas (2016, p. 40) la define como "la capacidad de un Gobierno de cumplir sus obligaciones de deuda, de manera indefinida, sin caer explícitamente en la insolvencia o en la cesación de pagos (default)".

Los hacedores de reglas fiscales parecen creer que cuando se logra la sostenibilidad fiscal el BC puede jugar un papel activo en busca del objetivo en la tasa de inflación sin tener en cuenta la posible presión fiscal dada por un aumento en la emisión de deuda, dado quela emisión de bonos públicos puede provocar que el BC no cumpla con dicho objetivo, pero, como afirman Bai y Leeper (2017, p. 105) "esta perspectiva parece malinterpretar la naturaleza de la determinación del nivel de precios"26, puesto que, no sólo son las decisiones de la autoridad monetaria las que importan para cumplir el objetivo de inflación, ni tampoco la sostenibilidad fiscal como herramienta de apoyo, sino que es el comportamiento fiscal pasivo el que proporciona el respaldo fiscal necesario para que la política monetaria funcione como lo dicta el EMI.

Esto se puede intuir por medio del siguiente ejemplo que exponen los autores:

Si el BC establece una política monetaria contractiva para disminuir la tasa de inflación surgen dos efectos:

1. Hay un incremento en la tasa de interés nominal, es decir, el rendimiento de los bonos aumenta.

2. La deuda asciende en términos nominales. 
Además, se supone que los agentes tienen en su poder bonos, si estos perciben que el aumento de la deuda no es contrarrestado por un alza en los impuestos o un aumento del superávit primario, ${ }^{27}$ supondrán que su nivel de riqueza es mayor debido al ascenso en la tasa de interés y como son poseedores de bonos tienen el incentivo de incrementar su demanda de bienes, a esto se le conoce como el efecto steepingon a rake enunciado por Sims (2011, p. 53). Básicamente este efecto señala que un aumento de la tasa de interés nominal en una primera instancia tiene un impacto positivo sobre la tasa de inflación disminuyéndola, pero, cuando el efecto riqueza por parte de los agentes se impone, la tasa de inflación se incrementa de nuevo y de manera sostenida de tal manera que resulta ser mayor que la inflación inicial. Lo anterior se muestra en el siguiente esquema:

$$
\uparrow i_{t} \rightarrow \downarrow \pi_{t} \rightarrow \uparrow \text { Efecto Riqueza } a_{t+1} \rightarrow \uparrow D_{t+1}^{\text {Bienes }} \rightarrow \uparrow \pi_{t+1}>\pi_{t}
$$

Por tanto, se puede señalar que la medida de política monetaria para disminuir la presión sobre la tasa de inflación en cierta forma parece ser la más eficaz en un principio, pero su análisis no está completo, debido a que no considera el papel de la política fiscal. La política monetaria ocasionará el efecto contrario en el siguiente período, cuestionando el análisis del EMI.

\subsection{Una propuesta de coordinación}

El objetivo de esta sección es analizar, retomando las ideas expuestas en el modelo propuesto por Bai y Leeper(2017) el comportamiento de la política fiscal. En principio se plantean los supuestos del modelo y posteriormente se procede con el análisis.

Supuestos del modelo:

1. Una economía cerrada y pequeña.

2. Dentro de la economía existe un agente representativo con un periodo de vida infinita.

3. El agente recibe una cantidad constante de bienes en cada periodo.

27 La Secretaria de Hacienda y Crédito Público (SHCP) define al superávit primario como la diferencia que existe entre los ingresos y gastos totales del Estado, sin considerar el pago por servicios de la deuda. 
4. El agente sólo obtiene utilidad del consumo de bienes.

5. La tasa de interés real de equilibrio es constante:

$$
R=\left(\frac{1}{\beta}-1\right) ; 0<\beta<1 ;
$$

Donde $R$ es la tasa de interés real y $\beta$ es el factor de descuento del consumidor. ${ }^{28}$ Dados los supuestos, Bai y Leeper proponen que el consumidor toma una decisión de consumo (y en consecuencia de ahorro) que está en función de la siguiente relación sencilla de Fisher:

$$
i_{t}=\beta \mathrm{E}_{\mathrm{t}}\left(\pi_{\mathrm{t}+1}\right)
$$

Definen $i_{t}$ como la tasa de interés nominal de los bonos del Estado comprados en $t$ y que se pagan en $t+1$, además funge como el instrumento de política monetaria, $\pi_{\mathrm{t}+1}$ es la tasa de inflación esperada en $t+1, E_{t}$ es el valor esperado.

Para deducir la ecuación (8), los autores imponen el equilibrio en la restricción del Estado:

$$
c_{t}=\gamma-g
$$

$c_{t}$ : es el consumo del Estado en el periodo, $\gamma$ es el ingreso constante del Estado y $g$ el gasto constante, de la ecuación (9) se deduce que el Estado compra una cantidad constante de bienes en cada periodo.

El modelo asume también una sencilla regla de política monetaria ${ }^{29}$ que es parecida a la expuesta en la sección anterior:

$$
i_{t}-i^{*}=\alpha\left(\pi_{t}-\pi^{*}\right) ; \alpha \geq 0
$$

Donde $i^{*}$ es la tasa de interés nominal acorde con el objetivo de inflación, también se podrá definir como $\frac{\pi^{*}}{\beta}$, quedando de la siguiente ma-
nera:

$$
i_{t}-\frac{\pi^{*}}{\beta}=\alpha\left(\pi_{t}-\pi^{*}\right) ; \alpha \geq 0
$$

28 Por factor de descuento debe entenderse al coeficiente que se utiliza para saber el valor presente de cualquier flujo de caja futuro.

29 Los autores plantean esta regla en términos de su inversa, pero nos parece que resulta más claro si la consideramos en estos términos con el fin de que sea comparable con la que propone Jones (2009) cuando explica el modelo de tres ecuaciones. 
En la ecuación (10) se mantiene lo explicado en la sección anterior; $\alpha$ es el parámetro de agresividad con el que el BC actúa ante desviaciones de la tasa de inflación efectiva con respecto a su objetivo, $\pi^{*}$ es la tasa de inflación objetivo. Esta regla relacionará el movimiento de la tasa de interés nominal en función de la desviación de la tasa de inflación efectiva con respecto a su objetivo. Es entonces que, cuando el parámetro $\alpha>\beta$, la política monetaria es activa y cuando el parámetro $\alpha>\beta$ la política monetaria es pasiva.

Hasta aquí, se puede observar el comportamiento del agente representativo, en función de la relación de Fisher, y el de la política monetaria que está en función del parámetro $\alpha$, el cual indica cómo debe comportarse la autoridad monetaria. Al inicio de este artículo señalamos que el EMI no hace explicito el papel de la política fiscal, el cual es fundamental para que se pueda cumplir el objetivo del EMI. A continuación, se verá cómo se comporta el Estado bajo este enfoque.

Los autores mencionan que a través de la política fiscal se recaudan impuestos de la economía $\left(\tau_{t}\right)$ y se asume que las compras son constantes $(g>0)$. El Estado también emite bonos en un periodo $t\left(B_{t}\right)$ que satisface su restricción:

$$
\frac{B_{t}}{P_{t}}+\tau_{t}=g+\frac{i_{t-1} B_{t-1}}{P_{t}}
$$

Donde $B_{t}$ son los bonos emitidos en t, $P_{t}$ es el nivel de precios agregado, $\tau_{t}$ es la recaudación de impuestos en el periodo $t$.

Antes señalamos que las reglas fiscales dan el soporte para conseguir la sostenibilidad fiscal, pero este comportamiento no necesariamente tiene que ir ligado con la pasividad fiscal, por lo que una regla fiscal que es comúnmente utilizada es:

$$
\tau_{t}-\tau^{*}=\gamma\left(\frac{B_{t-1}}{P_{t-1}}-b^{*}\right), \gamma \geq 0
$$

Donde $\tau^{*}$ es el nivel constante de recaudación de impuestos, $\gamma$ es un parámetro fiscal, $\frac{B_{t-1}}{P_{t-1}}$ es la deuda pública real de la economía del periodo t-1 y $b^{*}$ es el nivel constante de la deuda pública real. La ecuación (12) representa una regla de impuestos, la cual propone que la desviación de los 
impuestos debe ser proporcional a la desviación de la deuda pública real de largo plazo.

4.1.1. Estabilización y comportamiento fiscal pasivo

Los autores al combinar las ecuaciones (11) y (12) y al desarrollar la expresión, llegan a la siguiente ecuación:

$$
E_{t}\left(b_{T}-b^{*}\right)=\left(\frac{1}{\beta}-\gamma\right)^{T-t}\left(b_{t}-b^{*}\right) \text { donde } \mathrm{T}>\mathrm{t}
$$

La ecuación (13) marca la evolución de la deuda pública real a lo largo del tiempo. De esto se desprende, como los autores afirman, que cuando el parámetro fiscal $\gamma>0$ asegura que se espere que la deuda real $\left(b_{t}=\frac{B_{t}}{P_{t}}\right)$ aumente en menor proporción que $\frac{1}{\beta}$, además, con la regla de impuestos, representada por la ecuación (12) de la sección anterior, y cuando se asume al parámetro fiscal ${ }^{30}$ mayor a la tasa de interés real, es decir $\gamma>r$, se pueden observar dos cosas:

1. Cuando los impuestos son proporcionales al nivel de la deuda y a su vez son multiplicados por el parámetro fiscal que se asume mayor a la tasa de interés real, el aumento de la captación de ingresos por parte del Estado garantiza cubrir por una parte un aumento adicional en el servicio de la deuda ${ }^{31}$ y por otra amortizar la deuda recién emitida en cada periodo, dicho de otra forma, la deuda pública real se estabiliza y es acorde con el equilibrio de largo plazo.

2. A partir de la regla de impuestos representada por la ecuación (12), se observa una reacción de los impuestos ante cambios en el nivel de precios del periodo $t-1$.

\footnotetext{
30 En este modelo es clave el nivel del parámetro fiscal $(\gamma$ )a fin de cumplir con la condición de transversalidad (que garantiza que la senda de las variables no sea explosiva), la cual es suficiente y necesaria para la optimalidad de los hogares: $\lim _{T \rightarrow \infty} \beta^{T-t} E_{t} b_{t}$

Bohn (1998)señala que un criterio para saber si existe sostenibilidad fiscal son las pruebas que implican la estimación de una condición de transversalidad, la cual debe incorporar la deuda pública descontada con alguna tasa de interés. 31 Se define el servicio de la deuda como el pago de los intereses de la deuda generados, ya sea por capital o por algún préstamo concedido.
} 
Esto se puede verificar a partir del ejemplo expuesto al inicio de este apartado, donde el BC asume una política monetaria contractiva para disminuir la presión sobre la tasa de inflación.

Para que la política monetaria tenga efectos sobre la economía y asumiendo la regla de política fiscal, cuando el parámetro de política fiscal $\gamma>0$, muestra el comportamiento de la política fiscal para dar el respaldo necesario a la autoridad monetaria para que este pueda cumplir con su objetivo. En este caso, el BC asume que el nivel de precios del periodo t-1 es alto, por tanto, para el periodo t aumentará la tasa de interés para disminuir esta presión, pero al ascenso de la tasa de interés se traduce como un aumento en el rendimiento de bonos en el mismo periodo. Ante esto y para que los agentes no formen expectativas con relación al no actuar de la política fiscal y a su riqueza en el periodo $\mathrm{t}+1$, la política fiscal tendrá que subirlos impuestos para neutralizar el efecto riqueza de los agentes y por consiguiente que no haya un cambio en el patrón de consumo por parte de éstos que vuelva a provocar una presión sobre el nivel de precios en $\mathrm{t}+1$.

En consecuencia, para que el objetivo de estabilidad de precios se logre debe existir coordinación entre las dos políticas, ambas actuando de forma contractiva, donde la política monetaria tiene un papel activo en contra de la inflación y la política fiscal de forma pasiva brindando el respaldo necesario a la política monetaria para que esta sea eficaz, dicho de otra forma, la política fiscal está subordinada a la política monetaria.

\subsubsection{Estabilización versus comportamiento fiscal pasivo}

Aunque el mejor escenario para la economía es que exista estabilidad con relación a la deuda y pasividad en el comportamiento de la política fiscal, no necesariamente tienen que coincidir, esta sección trata de mostrar los diferentes escenarios expuestos por Bai y Leeper con relación a la existencia de estabilidad, pero no pasividad fiscal y cuando existe pasividad fiscal pero no estabilidad.

\section{Existencia de estabilidad de la deuda pública, sin comporta- miento fiscal pasivo:}

En este caso se asume estabilización, pero un comportamiento activo de la política fiscal. 
Se supone también que para cada periodo existe un superávit primario $(s>0)$, el cual se define como $s=\tau^{*}-g$. En este caso el parámetro fiscal $\gamma=0$, lo que implica que $\tau_{t}=\tau^{*}$ para cada periodo. Los autores al combinar el valor esperado con la ecuación (8)determinan el nivel constante de la deuda real, el cual es:

$$
b_{t}=b_{t-1}=b^{*}=\frac{\beta}{1-\beta} s ; 0<\beta<1 ;
$$

Al asumir un superávit primario constante en cada periodo, esta ecuación denota que el valor de la deuda pública real es constante en el largo plazo, por tanto, la política fiscal en este caso es sostenible, debido a que la deuda pública real es constante, además, al combinar la ecuación (14) con la restricción del Estado, los autores llegan a lo siguiente:

$$
\frac{i_{t-1} B_{t-1}}{P_{t}}=\frac{1}{1-\beta} s, 0<\beta<1 \text { y } s>0
$$

Esta ecuación implica que dados $i$ y $B$ en el periodo t-1, existe un único equilibrio con un nivel de precios positivo, siempre y cuando $s>0$, pero se puede observar que los impuestos no aparecen en la ecuación, esto implica que no reaccionan al nivel de precios, por tanto, la política fiscal no da el respaldo que tiene que brindar para que el objetivo del BC sea cumplido en su totalidad, también se puede observar que si la política monetaria aumenta la tasa de interés nominal en el periodo t, también subirá el servicio de la deuda en $t+1$, esto provocará que haya un ascenso en los ingresos de los agentes que no se podrán compensar con un aumento en los impuestos. Por lo tanto, la riqueza de los agentes es mayor, lo que conduce a que aumenten su demanda de bienes y por ende se generan presiones en el nivel de precios en el período $\mathrm{t}+1$. Entonces, si el superávit primario es constante en cada periodo, aunado a la política monetaria que usa una regla de política monetaria, esto tiende a producir un aumento sostenido en la tasa de inflación.

\section{Existencia de comportamiento fiscal pasivo con deuda públi- ca inestable}

En este caso, se asume que una economía puede tener un comportamiento fiscal pasivo, pero no hay condiciones para la estabilidad de la deuda pública real. Bai y Leeper (2017, p.107) sostienen que la política fiscal puede ser sostenible cuando la deuda pública relacionada al equilibrio 
cumple con las condiciones de transversalidad, pero en el caso en el que $R$ $>\gamma>0$ la deuda no es estable y su nivel aumenta a lo largo del tiempo. En el equilibrio se asume que el Estado necesita acceder a ingresos que puedan aumentar sin estar relacionados con el ritmo del aumento de la deuda, pero el equilibrio sólo se puede mantener cuando el Estado tiene acceso sin límites a los ingresos recaudados. Por lo tanto, cuando el pago de intereses sobre la deuda crece a una tasa $R-\gamma$, los ingresos de los poseedores de bonos también crecerán a la misma y si el Estado tiene acceso a ingresos que no modifican el patrón de consumo de los agentes, solamente estará extrayendo el aumento de los ingresos dados por los intereses para financiar el incremento de la deuda. Adicionalmente, si a la economía se le pusiera un tope de ingresos, es decir, una limitación en la recaudación de impuestos, entonces la deuda pública en algún momento va a aumentar más rápido que la economía. Por tanto, para evitar una situación de este tipo se tiene que cumplir que: $\gamma>R$, es decir, que el parámetro de política fiscal sea mayor que la tasa de interés real a fin de que el comportamiento fiscal pasivo también estabilice la deuda.

\section{Reflexiones finales}

Se hizo una breve revisión de las causas que llevaron a la política fiscal a ser segregada de los análisis en favor de la política monetaria como eje conductor de la política económica, lo cual se refleja en el NCM, donde las decisiones del $\mathrm{BC}$ en relación con el nivel de la tasa de interés nominal de corto plazo desempeñan un papel decisivo en la búsqueda de la estabilidad de precios.

¿El EMI es tan bueno cómo se cree? Roger (2010, p.49) plantea una serie de preguntas para saber si en verdad estos esquemas han sido tan efectivos como se piensa:¿Los EMI son eficaces cuando se tienen altas tasa de inflación? Esto debido a que en la gran mayoría de los países que han adoptado el EMI, su tasa de inflación no era tan elevada. En 2012 el promedio de tasa de inflación de adopción fue del 5.8\%, entonces es un poco ambivalente aseverar la efectividad de estos esquemas. ¿Qué tanto contribuyen los EMI al nivel de endeudamiento en las economías? Si los países siguen los criterios de sostenibilidad fiscal, y se tiene programado un cierto nivel de endeudamiento para un periodo, ¿qué tanto impactará una modificación en la política monetaria? 
Vimos que la política fiscal, aunque no esté de manera explícita en el marco del modelo de tres ecuaciones del NCM, si aparece de forma implícita, debido a que debe existir coordinación entre ambas políticas, tal como se deduce del trabajo de Bai y Leeper (2017), donde la política fiscal debe brindar un soporte pasivo a la política monetaria en función de los objetivos que esta se proponga para estabilizar la tasa de inflación. La política fiscal debe neutralizar los efectos producidos por el movimiento en la tasa de interés para que la autoridad monetaria cumpla con el objetivo de estabilidad de precios. Adicionalmente, la política fiscal debe de mantener estabilidad en la deuda pública, para lo cual se tiene que sujetar a una regla de impuestos que mantenga el equilibrio presupuestal intertemporal.

Una de las principales preocupaciones tanto para economías industrializadas como para países emergentes es el problema de la nueva deuda, ya que se observa un incremento en sus niveles de deuda en relación con el producto interno bruto, lo cual cuestiona la sustentabilidad fiscal en sus distintas definiciones. En 2016 la deuda mundial se encontraba en máximos históricos alcanzando un nivel de 164 billones de dólares, equivalente al 225 \% del PIB mundial. En las economías emergentes y de ingreso medio, la deuda registra un nivel promedio cercano al 50\% del PIB. ${ }^{32}$

En el caso del modelo desarrollado por Bai y Leeper (2017) la situación anterior implica que el parámetro fiscal es menor a la tasa de interés real, lo que en el largo plazo genera que la deuda no sea estable, puesto que su nivel está aumentando. Por lo tanto, ya no se verifica la condición de transversalidad y en consecuencia no existe sustentabilidad fiscal.

Obviamente el problema de la deuda no es exclusivo de los países que aplican EMI, en el ascenso de la deuda incide una gran cantidad de factores que tienen que ver tanto con el ingreso como con el gasto presupuestal. En este trabajo se planteó que en las economías que aplican EMI no se puede dejar de lado el papel de la política fiscal, y lo que se observa es que hay una tendencia a incurrir cada vez en déficits mayores, lo que arroja una pregunta que genera controversia en torno a las políticas fiscales expansivas financiadas con déficit, ¿hasta qué grado conviene a una economía endeudarse? Si bien es cierto que el trabajo de Reinhart y Rogoff (2010)

32 Véase Monitor Fiscal del Fondo Monetario Internacional, abril 2018. 
contiene omisiones en su muestra estadística evidenciadas por Herdon y otros (2014), de ambos trabajos se puede deducir la existencia de un cierto grado de correlación entre deuda y crecimiento. La Congressional Budget Office, en 2002 realizó un estudio sobre la evolución del déficit y el gasto público como porcentaje del PIB en Estados Unidos, asumiendo que no haya cambios en su política hasta 2075; de acuerdo a este estudio, se prevé que el déficit aumente de forma exponencial hasta alcanzar un 20.7\% del PIB, cuando en 1950 este indicador sólo representaba 1.1\% y el gasto para 2075 representará un $40 \%$ como porcentaje del PIB, concluyendo que este tipo de política es insostenible si se sigue manteniendo la política actual.

Finalmente, en el libro coordinado por Blanchard y otros, títulado Progress and Confusion: The State of Macroeconomic Policy de 2016, se discuten cuestiones relacionadas con el papel de la política fiscal en el futuro. Consideran que el campo de las finanzas públicas ha sido prolífico a la hora de analizar una serie de ámbitos en los que el Estado interviene o debería intervenir. Observan que la capacidad del Estado para tributar con el fin de sostener la expansión de los presupuestos se encuentra bajo presión en el siglo XXI. Las finanzas públicas están siendo moldeadas por dinámicas evolutivas como la globalización, el cambio tecnológico, las transiciones demográficas, la integración regional y muchas otras. Gaspar (2016) centra su interés en aspectos relacionados con las finanzas públicas, la estabilidad macroeconómica y el crecimiento. Por otro lado, Buti (2016) aborda la pregunta de si la política fiscal puede estimular la inversión sin los riesgos financieros asociados a la política monetaria de expansión cuantitativa aplicada por los países desarrollados ante la crisis financiera mundial de 2008-2009. Finalmente, De Long (2016) se plantea la pregunta: ¿cuál es el futuro de la política fiscal basada en reglas? Considera que al igual que en el ámbito de la política monetaria, la política fiscal basada en normas pone de manifiesto una serie de restricciones.

\section{Referencias Bibliográficas}

Arenas de Mesa, A. (2016).Sostenibilidad fiscal y reformas tributarias en América Latina, Santiago: Comisión Económica para América Latina y el Caribe.262p.

Bai, Y., y Leeper, E. M. (2017). Fiscal stabilization vs. passivity.Economics Letters, 154(Supplement C), p. 105-108. 
Barro, R. J. (1974). Are Government Bonds Net Wealth? Journal of Political Economy, 82(6), p. 1095-1117.

Bernanke, B. S.; Laubach, T.; Mishkin, F. S., y Posen, A. S. (2001). Inflation Targeting: Lessons from the International Experience: Princeton University Press. 382p.

Blaug, M. (2001). Teoría Económica en Retrospección (E. L. S. Galindo, Trans.): Fondo de Cultura Económica.819p.

Blinder, A. S. (2000). Central-Bank Credibility: Why Do We Care? How Do We Build It? The American Economic Review, Vol. 90, No. 5, p. 1421-1431.

Bohn, H. (1998). The Behavior of U.S. Public Debt and Deficits. Quarterly Journal of Economics, Vol. 113, No. 3, p. 949-963.

Buti, M. (2016). What Future for Rules-Based Fiscal Policy? In O. Blanchard y otros (Ed.), Progress and Confusion. The State of Macroeconomic Policy (pp. 183-96). London, England: The MIT Press.

Clarida, R.; Jordi Gali y M. Gertler (1999). “The Science of Monetary Policy: A New Keynesian Perspective".Journal of Economic Literature, 37(4), p.1661-1707.

Congressional Budget Office (2002). A 125-year Picture of the Federal Government's Share of the Economy, 1950 to 2075, Vol. 1: Long-Range Fiscal Policy Brief, p. 1-4.

DeLong, J. B. On the Proper Size of the Public Sector and the Level of Public Debt in the Twenty-First Century. In O. Blanchard y otros (Ed.), Progress and Confusion. The State of Macroeconomic Policy (pp. 197-214). London, England: The MIT Press.

García, L, A. y Perrotini, I. (2014). Modus Operandi del Nuevo Consenso Macroeconómico en Brasil, Chile y México.Problemas del Desarrollo, Vol. 45, No. 179 , p. 35-63.

Gaspar. V. (2016). Fiscal Policy for the Twenty-First Century: Testing the Limits of the Tax State? In O. Blanchard y otros (Ed.), Progress and Confusion. The State of Macroeconomic Policy (pp. 165-76). London, England: The MIT Press.

Hanel, C. M., y Rosenthal, M. S. (1997). Metas de inflación como instrumento de política monetaria. México: Banco de México.

Herndon, T.; Ash, M., y Pollin, R. (2014). Does high public debt consistently stifle economic growth? A critique of Reinhart and Rogoff. Cambridge Journal of Economics, 38(2), p. 257-279.

Hetzel, R. L. (2004). How do central banks control inflation?.Economic Quarterly(Sum), Vol. 90, No. 3, p. 46-63. 
Jones, C. I. (2009). Macroeconomía (E. Rabasco\& L. Toharia, Trans.): Antoni Bosch Editor, 706p.

Keynes, J. M. (1936). Teoría general de la ocupación, el interés y el dinero (2014, $4^{a}$ ed.). México: Fondo de Cultura Económica. 413p.

Kydland, F. E., y Prescott, E. C. (1977). Rules Rather Than Discretion: The Inconsistency of Optimal Plans. Journal of Political Economy, 85(3), p. 473-491.

Landreth, H., y Colander, D. C. (2006). Historia del pensamiento económico: McGraw-Hill Interamericana de España.546p.

Leeper, E. M. (1991). Equilibria under 'active' and 'passive' monetary and fiscal policies.Journal of Monetary Economics, Vol. 27, No. 1, p. 129-147.

Lerner, A. P. (1944). The Economics of Control: Principles of Welfare Economics: Macmillan, New York, 428p.

Lucas, R. (1972). Expectations and the neutrality of money. Journal of Economic Theory, 4(2), p. 103-124.

Mankiw G. y D. Romer (1991). New Keynesian Economics, Vol. 1: Imperfect Competition and Sticky Prices 430p; y II: Coordination Failures and Real Rigidities 450p., edited by The MIT Press, London, England.

Meulendyke, A. M. (1988). A Review of Federal Reserve Policy Targets and Operating Guides in Recent Decades. Federal Reserve Bank of New York.Quarterly Review, p. 6-17.

Reinhart, C., y Rogoff, K. (2010). Growth in a Time of Debt. American Economic Review, 100(2), p. 573-578.

Roger, S. (2010). Inflation Targeting Turns 20. Finance and Development, 47, p. 46-49.

Sims, C. A. (2011). Stepping on a rake: The role of fiscal policy in the inflation of the 1970s.European Economic Review, Vol. 55, No. 1, p. 48-56.

Taylor, John B. (1993). Discretion versus Policy Rules in Practice, Carnegie-Rochester Conference Series on Public Policy 39, p. 195-214.

Snowdon, B., y Vane, H. R. (2002). An Encyclopedia of Macroeconomics. E. Elgar, $721 \mathrm{p}$.

Walsh, C. E. (2008). Central bank independence. In S. N. Durlauf \& L. E. Blume (Eds.), The New Palgrave Dictionary of Economics. Basingstoke: PalgraveMacmillan. 